\title{
Inhibition of return in manual and saccadic response systems
}

\author{
KEVIN A. BRIAND, ABIGAIL L. LARRISON, and ANNE B. SERENO \\ Rutgers University, Newark, New Jersey
}

\begin{abstract}
When nonpredictive exogenous visual cues are used to reflexively orient covert visual spatial attention, the initial early facilitation for detecting stimuli at cued versus uncued spatial locations develops into inhibition by $300 \mathrm{msec}$ following the cue, a pattern referred to as inhibition of retum (IOR). Experiments were carried out comparing the magnitude and time course for development of IOR effects when manual versus saccadic responses were required. The results showed that both manual and saccadic responses result in equivalent amounts of facilitation following initial exposure to a spatial cue. However, IOR developed more quickly for saccadic responses, such that, at certain cue-target SOAs, saccadic responses to targets were inhibited, whereas manual responses were still facilitated. The findings are interpreted in terms of a premotor theory of visual attention.
\end{abstract}

Inhibition of return (IOR) was first reported by Posner and Cohen (1980, 1984; see Klein, 2000, for a recent review). The most commonly accepted explanation for IOR is that some mechanism prevents spatial attention from returning to recently sampled positions, thus increasing the efficiency of visual search in complex environments (e.g., Abrams \& Dobkin, 1994; Posner \& Cohen, 1984; Pratt, Kingstone, \& Khoe, 1997; Rafal, Calabresi, Brennan, \& Sciolto, 1989; Rafal, Egly, \& Rhodes, 1994). It is as yet unclear, however, how best to characterize the nature of the spatial attention system underlying this effect.

According to a frequently proposed mechanism for IOR (that we will refer to as the standard model of IOR), spatial attention operates via some sort of a map or representation of visual space. Stimuli are selected for further processing by reference to this spatial map. Presumably, spatial attention somehow enhances entry of information into higher level processes that determine what responses are to be made to stimuli. The poorer processing of stimuli observed in IOR occurs because attention cannot easily be allocated to previously selected locations. If stimuli occur at these particular locations, higher level processes will have inhibited access to information concerning them. An implicit (and perhaps explicit) assumption of this approach is that what is to be done with the stimulus (i.e., the response required) has no bearing on spatial cuing

This work was supported in part by grants from the National Alliance for Research in Schizophrenia and Depression (NARSAD) and the Scottish Rite Foundation to K.A.B. and by NARSAD, McDonnell-Pew, and the Scottish Rite Foundation to A.B.S. The authors thank Todd Horowitz and two anonymous reviewers for comments and suggestions during manuscript preparation. Correspondence should be addressed to K. A. Briand, Department of Neurobiology and Anatomy, University of Texas-Houston Medical School, 6431 Fannin St., Houston, TX 77030 (e-mail: kevin.a.briand@uth.tmc.edu). and IOR effects. Both spatial cuing and IOR are primarily or wholly related to input or stimulus selection processes.

A central aspect of this approach is that the neural system (or systems) underlying spatial attention are both functionally and structurally separate from the system (or systems) underlying data processing. IOR is assumed to be associated with an inhibition in the operation of the attention process itself (e.g., Klein, 1988; Posner, Rafal, Choate, \& Vaughan, 1985). This general viewpoint holds even in studies that have examined other aspects of IOR, including whether it can be obtained in auditory covert orienting (Mondor, Breau, \& Milliken, 1998) and whether IOR can be object-based as well as location-based (Abrams \& Dobkin, 1994; Gibson \& Egeth 1994; Jordan \& Tipper, 1998; Tipper, Driver, \& Weaver, 1991; Tipper, Jordan, \& Weaver, 1999; Tipper, Weaver, Jerreat, \& Burak, 1994).

Despite the prominence of this approach in the literature, an alternative explanation has persisted, which assumes instead that IOR is associated with stimulus and response processes (Klein \& Taylor, 1994; Rizzolatti, Riggio, Dascola, \& Umiltà, 1987). Much of the impetus for this view of spatial attention and IOR comes from the fact that many studies have found a close relationship between spatial attention and saccadic eye movements ${ }^{1}$ (Kustov \& Robinson, 1996; Rafal et al., 1989; Shepard, Findlay, \& Hockey, 1986; see also Sereno, 1996).

The most prominent single model subscribing to this view is "premotor theory." The core assumption of this approach is that the system that controls action is the same as what we call spatial attention (Rizzolatti, Riggio, \& Sheliga, 1994). More precisely, the claim of premotor theory is that the physiological maps used to represent visual space not only code the locations of objects but are also involved in motor programming. What we call "spatial attention" is the consequence of the activity in spatial maps and is related both to sensory processing and to motor preparation. In short, we attend to spatial locations to 
which we are preparing to make some type of response (e.g., saccade or reaching movement). The act of attending to a spatial location is nothing more or less than the act of preparing to make some type of response to that location.

Rizzolatti et al. (1994) summarize the evidence in favor of such an approach. First, there is no known single spatial map on which a separable attention system could operate. Rather, there appear to be a number of frontal and parietal areas that code space. Second, these different cortical areas do not code space per se but instead appear to code properties of space relevant for specific types of actions. For example, neurons in a circuit involving the lateral intraparietal area (LIP) and the frontal eye fields (FEF) code space retinotopically, firing when a saccade is being prepared to their receptive field. However, neurons in a parietal (area 7b)-premotor (area F4) circuit code space in a body-centered frame of reference and fire only when a reaching movement to the receptive field is to be executed and not when a saccade to that location is planned. Furthermore, these neurons have receptive fields that are restricted to peripersonal space (i.e., they are active only for stimuli or spatial locations that are reachable). The general (and neurophysiologically well-founded) assumption here is that the dorsal cortical pathway usually considered as responsible for spatial perception or "where" processing (Ungerleider \& Mishkin, 1982) is instead best viewed as coding for action in space (i.e., not "where" but "how"; see Goodale \& Milner, 1992). Furthermore, different types of motor actions require unique spatiomotor submaps, a fact that may have implications for the mechanisms underlying IOR.

These two general explanations of spatial cuing and IOR effects continue to have their proponents and critics. One simple manipulation that may provide data relevant to distinguishing between them would be to examine the time course of spatial cuing and IOR effects for different response systems (e.g., eye movements vs. manual responses). The rationale for this strategy follows directly from the different assumptions of the standard model and premotor theory. Under the standard model of spatial attention, there is a single spatial representation that is accessed by a separate attention system. Regardless of whether the "inhibition" in IOR is a property of the spatial map or the attention system that accesses this map, it should not matter what the actual response to a stimulus is. If the task requires localization of the target, for example, it should not matter whether localization is accomplished via saccades to the target or by some type of manual response. Thus, the standard model would not predict any effect of response mode on the time course or magnitude of IOR.

On the other hand, according to premotor theory the act of attending to a point in space can be best understood as the consequence of activity within the various cortical maps used to represent space. Since the spatiomotor maps used to code for saccadic and manual responses are functionally separate, there is no reason to assume that they would operate in exactly the same way or with an identical time course. Thus, if premotor theory is correct, one might expect that the particular response (e.g., manual vs. saccadic) could affect the characteristics of the IOR obtained. If the magnitude or, in particular, the time course of IOR differs for saccadic and manual versions of the same task, this finding would be more easily accounted for by a premotor or response-based model of spatial attention.

A comparison of manual and saccadic response systems in an IOR paradigm with reflexive cues has yet to be extensively investigated. An early study by Maylor (1985) directly compared manual and saccadic responses following reflexive cues and reported that they did not give equivalent effects. Manual responses resulted in early facilitation and late inhibition (the usual IOR effect), whereas saccadic responses showed no early facilitation, but, at longer SOAs, there was enhanced inhibition (relative to that found for manual responses).

Maylor's (1985) report is suggestive of a dissociation between IOR for manual and saccadic response systems. However, she never showed facilitation in the saccadic paradigm, hence raising the question of whether attention was first drawn to the cued location. Another study (Reuter-Lorenz, Jha, \& Rosenquist, 1996) included a comparison of manual and saccadic responses as part of its investigation of IOR but used only long cue-target stimulus onset asynchronies (SOAs; 1,000 and $1,300 \mathrm{msec}$ ) and thus can shed no light on their relative time course. Parenthetically, Reuter-Lorenz et al. (1996) found no IOR differences between manual and saccadic responses. Finally, a study by Shimojo, Tanaka, Hikosaka, and Miyauchi (1996) also compared manual and saccadic responses in an IOR paradigm. These investigators varied the time interval between the response to one stimulus and the onset of the next stimulus (from 1,000 to $1,800 \mathrm{msec}$ ). They tested only a range of longer interstimulus intervals (ISIs), much longer than that used by Reuter-Lorenz et al. (1996). Furthermore, it was unclear from their report what the relative magnitude of IOR was between the two response modes.

We propose that Maylor's (1985) analysis of manual versus saccadic IOR paradigms merited revisitation for a number of reasons. First and foremost, the premotor theory of visual attention (Rizzolatti et al., 1994) raises the possibility that rather fundamental differences might exist between manual and saccadic response systems in spatial orienting tasks, yet Maylor's study is the only one in the literature that investigated this issue. Besides this factor, two aspects of the procedures Maylor used suggest the need for further study: (1) the effects of the cuing procedure Maylor used needed to be examined in more detail, and (2) the range of cue-target SOAs she used to assess time course was limited. The present experiments were designed to make a more detailed comparison of both the magnitude and the time course of facilitation and IOR as it develops within manual and saccadic paradigms. 


\section{Cue-Back}

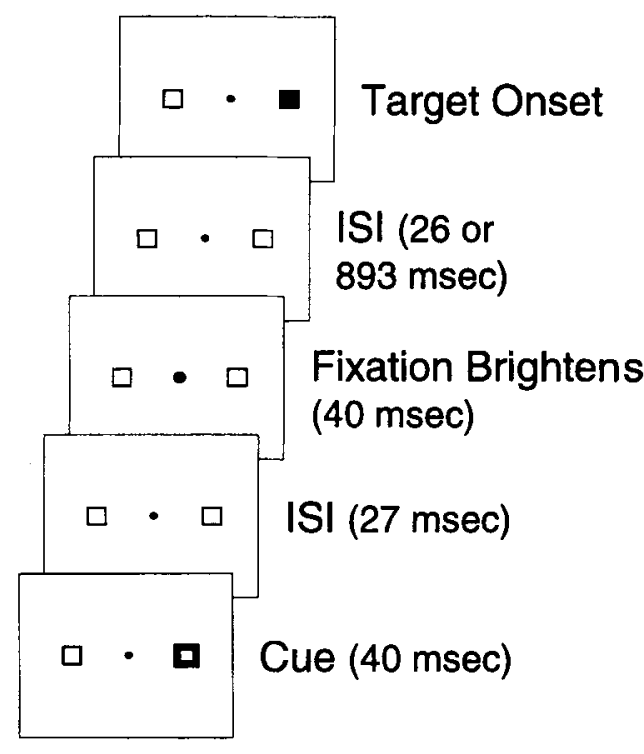

\section{One-Cue}

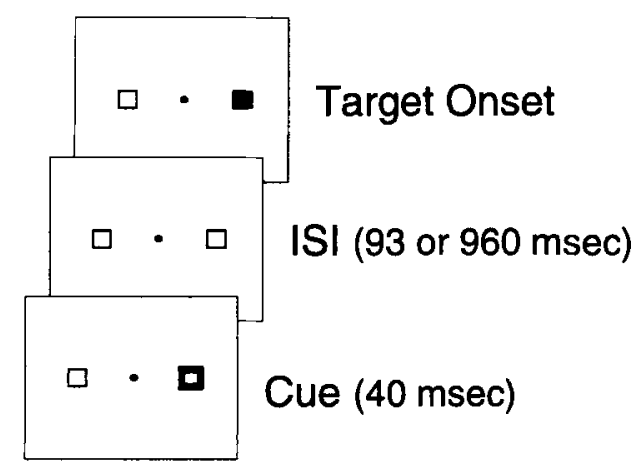

Figure 1. Sequence of stimulus events used in the cue-back and one-cue conditions of Experiment 1.

\section{EXPERIMENT 1}

Experiment 1 was a control experiment involving only saccadic responses. Maylor (1985) used a visual cue composed of two events, one of which was a brightening of a peripheral box for $100 \mathrm{msec}$, followed by the temporary brightening of a fixation point. This "cue-back" procedure may have caused the elimination of early facilitation for saccadic responses. It is reasonable to assume that the cuing procedure used is an important determinant of both the magnitude and the time course of development of facilitation and IOR. We thus directly compared a cue-back procedure with one wherein only a single peripheral visual cue was used.

\section{Method}

Subjects. Eighteen subjects were tested in a control experiment involving only saccadic responses. All subjects were undergraduates at Rutgers, Newark, received either partial course credit or monetary compensation for participating, and had normal or corrected vision.

Apparatus and Stimuli. Eye movements were recorded using an ISCAN RK-426 eye-tracking system, interfaced with an infrared sensitive camera. Spatial resolution was approximately $0.5^{\circ}$ of visual angle; temporal resolution to detect saccades was set at $16 \mathrm{msec}$. The subject placed his/her head on a chinrest positioned $72 \mathrm{~cm}$ from a computer monitor used to display the stimuli. The stimulus display consisted of a gray fixation spot $\left(0.2^{\circ} \times 0.2^{\circ}\right)$ on a black background, flanked by two gray boxes $\left(1.0^{\circ} \times 1.0^{\circ}\right)$ positioned such that their centers were $4.8^{\circ}$ to the left and right of fixation. The target stimulus was a green square measuring $0.6^{\circ} \times 0.6^{\circ}$ that appeared in the center of one of the two flanking boxes. Timing of stimuli durations and SOAs was accomplished by synchronizing stimulus displays with the refresh rate of the display monitor
(Sony Trinitron Multiscan 17sf II). The monitor had a refresh rate of $75 \mathrm{~Hz}$; thus, every time period used for display purposes was a multiple of $13.33 \mathrm{msec}$. The stimulus durations reported below are rounded to the nearest millisecond.

Procedure. Figure 1 shows examples of the stimulus display and the sequence of events for the cue-back and one-cue conditions. Each subject was tested in both conditions, given in separate blocks of 80 trials, or 20 trials each for the factorial combination of SOA (133 or $1,000 \mathrm{msec}$ ) and cue position (cued or uncued). The order of the two blocks was counterbalanced across subjects. Trial type was determined randomly within each block. A practice block of 16 trials was given to the subjects prior to each experimental block to familiarize them with the task and with the operation of the eyetracking system.

Cue-back. The subjects fixated the central fixation spot to initiate the trial sequence. If they successfully maintained fixation for $1,000 \mathrm{msec}$, the sequence of cue and target events was initiated. However, if their estimated point of gaze shifted more than $2.2^{\circ}$ from the center of the fixation point, the trial was canceled and placed back in the pool of uncompleted trials.

After the fixation period expired, there was a 40-msec brightening of one of the two peripheral boxes, which acted as a cue. After an additional $27-\mathrm{msec}$ period elapsed, the fixation point itself then brightened for $40 \mathrm{msec}$. The entire duration of the cue sequence, from initial brightening of the box to termination of the fixation brightening, was $107 \mathrm{msec}$. Following the cue, the fixation screen was displayed for either 26 or $893 \mathrm{msec}$ before target onset, resulting in effective SOAs between the initial cue and the target of 133 and 1,000 msec. The subjects had to make a speeded saccade to the target following its appearance, and the target remained in view until the subjects had completed a saccade to one of the two boxes. Brightening of the cue displays was accomplished by switching the color of the box and fixation point from gray to white.

There were two types of trials, defined by what preceded target onset. On cued trials, the target was shown within the peripheral box that had brightened. On uncued trials, the target was shown within the box that had not brightened. The probability of the tar- 
Table 1

Mean Response Times (in Milliseconds) and Percentage

Errors for Saccades in Experiment 1 as a Function of Cue Type (Cue-Back or One-Cue), Cue Position, and SOA

\begin{tabular}{|c|c|c|c|c|c|c|c|}
\hline \multirow[b]{2}{*}{ Cue Type } & \multirow{2}{*}{$\begin{array}{c}\text { SOA } \\
(\mathrm{msec})\end{array}$} & \multicolumn{2}{|c|}{ Cued } & \multicolumn{2}{|c|}{ Uncued } & \multicolumn{2}{|c|}{ Uncued - Cued } \\
\hline & & $\mathrm{RT}$ & $\%$ Error & RT & $\%$ Error & RT & $\%$ Error \\
\hline \multirow[t]{2}{*}{ Cue-Back } & 133 & 375 & 1.4 & 364 & 3.4 & -11 & $2.0^{*}$ \\
\hline & 1,000 & 392 & 1.4 & 353 & 0.7 & $-39 t$ & -0.7 \\
\hline \multirow[t]{2}{*}{ One-Cue } & 133 & 350 & 1.7 & 377 & 5.7 & $27 \dagger$ & $4.0_{+}^{+}$ \\
\hline & 1,000 & 411 & 1.2 & 368 & 0.0 & $-43 t$ & -1.2 \\
\hline
\end{tabular}

${ }^{*} p<.07 . \quad{ }^{\dagger} p<.01 . \quad{ }^{\ddagger} p<.001$.

get appearing within the brightened box was 50:50; hence, cues were unpredictive of target location. Saccade latency and accuracy were recorded using the signal provided by the eye tracker.

One-cue. The sequence of events is also shown in Figure 1. It was essentially the same as that for the cue-back procedure, with the exception that the fixation spot did not brighten following the brightening of the peripheral cue. The SOAs between cue and target were again 133 and $1,000 \mathrm{msec}$.

Following target onset, saccade latency was calculated as the elapsed time until the subject first moved his/her eyes beyond $2.2^{\circ}$ from the fixation spot. The end of the saccade was indicated when the subject's eyes were within $2.2^{\circ}$ of the center of either of the peripheral boxes. Correct responses were saccades that terminated at the window located where the target appeared. Saccades that terminated in any other position on the screen were coded as errors. If a successful saccade was not made within $1,000 \mathrm{msec}$ of target onset, a tone was sounded to indicate nonresponse, and that trial was replaced in the pool of unfinished trials to be completed later. The target remained in view until the saccade was completed.

\section{Results}

Trials with response times (RTs) longer than $700 \mathrm{msec}$ or faster than $80 \mathrm{msec}$ and trials in which blinks occurred were excluded from analysis (less than $1.0 \%$ of all trials). Each subject's mean correct RT for cued and uncued trials at the short (133-msec) and long $(1,000-\mathrm{msec})$ SOAs was calculated separately for the cue-back and one-cue conditions (see Table 1). Error rates (saccades to nontarget locations) were similarly calculated.

RT. As can be seen in Table 1, the one-cue condition showed the typical time course pattern of early facilitation followed by IOR at longer SOAs. However this was not the case for the cue-back condition, which showed only the later IOR effect. An analysis of variance (ANOVA) was executed, using the factors of cue format (cue-back or one-cue), cued-uncued, and SOA. The only significant main effect was that of cued-uncued $[F(1,17)=18.80$, $p<.001$ (faster RTs on uncued trials)]. The SOA effect was marginal $[F(1,17)=3.95, p<.07$ (faster RTs at the short SOA)]. All of the two-way interactions were significant or marginal [cue format $\times$ SOA, $F(1,17)=4.71, p<$ .05 (an effect of SOA in the one-cue condition but not in the cue-back condition); cue format $\times$ cued-uncued, $F(1,17)=10.59, p<.005$ (larger cuing effect in the cueback condition); SOA $\times$ cued-uncued, $F(1,17)=89.07$, $p<.0001$ (facilitation at the short SOA and IOR at the long SOA)]. Finally, the three-way interaction between cue format, cued-uncued, and SOA was also significant $[F(1,17)=7.57, p<.02]$.

To clarify the nature of this higher order interaction, planned contrasts were used to assess the size of the cuing effect (uncued RT - cued RT) for all SOA and cue format combinations. In the one-cue condition, there was facilitation [27 msec; $F(1,17)=12.91, p<.003]$ at the short SOA; however, this developed into inhibition at the long SOA $[-43 \mathrm{msec} ; F(1,17)=30.90, p<.001]$. In the cueback condition, the inhibition was not significant at the short SOA [ $-11 \mathrm{msec} ; F(1,17)=2.12, p>.16]$ but was significant at the long SOA $[-39 \mathrm{msec} ; F(1,17)=26.06$, $p<.001]$.

Errors. A similar analysis was conducted on percent error rates. There were only two significant effects. First, there was a main effect of SOA $[F(1,17)=21.25, p<$ $.0002]$, with higher error rates at the short SOA $(3.1 \%$ vs. $0.8 \%)$. Second, the interaction between SOA and cueduncued was significant $[F(1,17)=12.79, p<.003]$. This interaction shows that, at the short SOA, error rates were lower at cued versus uncued locations ( $1.6 \%$ vs. $4.5 \%)$, whereas, at the long SOA, the trend went in the opposite direction $(1.3 \%$ vs. $0.4 \%)$. The higher order interaction of cue format, cued-uncued, and SOA was not significant $[F(1,17)=1.65, p>.21]$.

Contrasts on the cuing effect for errors were also conducted (see Table 1). In the one-cue condition, there was facilitation at the short $\operatorname{SOA}[F(1,17)=16.17, p<.001$ (i.e., more errors on uncued trials)], whereas there was no cuing effect at the long $\operatorname{SOA}[F(1,17)=1.37, p>.25]$. In the cue-back condition, facilitation was marginally significant at the short SOA $[F(1,17)=3.86, p<.07]$, whereas there again was no cuing effect at the long SOA $[F(1,17)<1]$.

\section{Discussion}

The results of Experiment 1 confirm that the cuing paradigm (cue-back vs. one-cue) does affect the magnitude and the time course of facilitation and IOR. When a simple peripheral cue is used and no attempt is made to explicitly remove attention from the cued location, there is early facilitation at cued positions, which develops into IOR by an SOA of $1,000 \mathrm{msec}$. However, if attention is first drawn to and then immediately removed from a visual location, saccadic responses are no longer facilitated at an SOA of $133 \mathrm{msec}$ after the appearance of the cue and are nonsignificantly inhibited. Although the early nonsignificant inhibition displayed in the cue-back condition is complicated by the fact that an opposite and significant effect occurred for errors (i.e., faster saccade latencies on uncued trials were associated with significantly higher error rates), it remains clear that removing attention from the cued position dramatically affects the typical pattern of significant facilitation at short cue-target SOAs. We believe that the failure to observe facilitation in the cue-back condition is because the cue-back procedure accelerates the onset of IOR. Hence, any lingering 


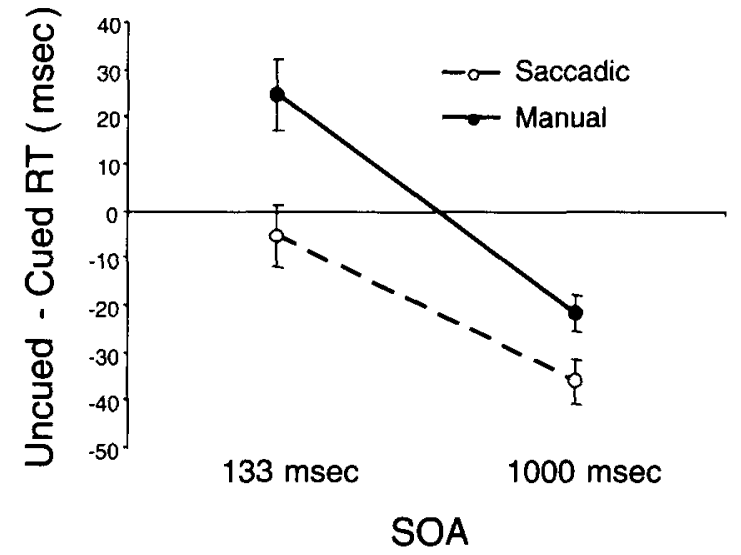

Figure 2. Experiment 2, cuing effect (uncued RT - cued RT) as a function of SOA and response mode (saccadic or manual).

facilitatory effects of the cue are eliminated by the time the target appears $133 \mathrm{msec}$ later.

Given that we were interested in examining the time course of IOR under different response conditions, we used a cue-back procedure in the remaining experiments reported here because we believed it more precisely and reflexively controlled the movement of attention. Furthermore, the use of a cue-back procedure also allowed us to make a more direct comparison with the results originally reported by Maylor (1985), since that is the procedure she employed.

\section{EXPERIMENT 2}

Having shown that the cue-back procedure can eliminate early facilitatory effects of peripheral cues on saccadic responses, the next step was to directly compare cuing effects in saccadic and manual versions of this task. Experiment 2 used the same procedures as in Experiment 1 except that either manual or saccadic localization responses were made.

\section{Method}

Subjects. Two groups of 23 subjects were tested. One group performed the IOR task using a manual response to indicate target position; another group performed the task using saccades to localize targets, as in Experiment 1.

Procedure. The procedure for the saccadic task was identical to that for the cue-back condition in Experiment 1. In the manual task, eye position was not monitored, and the subjects used a mouse to control the position of a cursor shown on the display screen. When the subjects wished to initiate a trial, they moved the cursor to the position of the fixation spot, and the sequence of stimulus events was identical to that for the saccadic condition. Instead of using point of gaze to determine when a saccade started and ended, the manual version of the IOR task kept track of where the cursor was positioned. For example, once a trial was initiated, the subject had to keep the cursor at the fixation spot for $1,000 \mathrm{msec}$ before the stimulus sequence was allowed to proceed. The radius of the window around the fixation or target areas used to determine the start and end of manual responses was identical with that used in the saccadic version of the task (e.g., a manual response was assumed to have started as soon as the cursor moved $2.2^{\circ}$ from the fixation point). Each subject was tested in a single block of 80 trials, preceded by a practice block of 16 trials.

\section{Results}

RT. Trimming of data (e.g., blinks, anticipations, and slow or fast responses) resulted in exclusion of only $2.9 \%$ of the data. As before, each subject's mean RT and percentage of erroneous responses from the remaining trials were calculated for each condition. The results (Figure 2) are plotted as difference scores (uncued RT - cued RT), with positive values indicating facilitation and negative values indicating the presence of IOR. Table 2 provides the statistics concerning the individual cuing effects.

As can be seen in Table 2 and Figure 2, the manual and saccadic versions of this localization task showed a different time course for cuing effects. Manual responses were initially facilitated at cued positions and then were inhibited, whereas saccadic responses showed no early facilitation but did show later IOR. An ANOVA combining the factors of response (manual or saccadic), SOA, and cued-uncued was executed, and there were significant main effects of response $[F(1,44)=54.45, p<.0001(449$

Table 2

Mean Response Times (in Milliseconds), Percent Errors, and Cue Position Effects (Uncued - Cued) in Experiment 2 as a Function of Response Type and SOA, Along With the $F$ Values for Planned Contrasts Used to Assess Cuing Effects and the Probability $(p)$ Levels Associated With Those Particular Contrasts

\begin{tabular}{|c|c|c|c|c|c|c|c|c|c|c|}
\hline \multirow{3}{*}{$\begin{array}{c}\text { SOA } \\
\text { (msec) }\end{array}$} & \multirow{2}{*}{\multicolumn{2}{|c|}{ Cued }} & \multirow{2}{*}{\multicolumn{2}{|c|}{ Uncued }} & \multirow{2}{*}{\multicolumn{2}{|c|}{ Uncued - Cued }} & \multicolumn{4}{|c|}{ Planned Contrasts } \\
\hline & & & & & & & \multicolumn{2}{|c|}{$\mathrm{RT}$} & \multicolumn{2}{|c|}{$\%$ Error } \\
\hline & RT & $\%$ Error & RT & $\%$ Error & RT & $\%$ Error & $F$ & $p<$ & $F$ & $p<$ \\
\hline \multicolumn{11}{|c|}{ Saccadic Responses } \\
\hline 133 & 372 & 1.1 & 367 & 2.6 & -5 & 1.5 & 0.96 & .34 & 7.93 & .008 \\
\hline 1,000 & 397 & 1.1 & 361 & 0.4 & -36 & -0.7 & 43.40 & .001 & 1.57 & .22 \\
\hline \multicolumn{11}{|c|}{ Manual Responses } \\
\hline 133 & 443 & 0.0 & 467 & 0.6 & 24 & 0.6 & 19.97 & .001 & 1.17 & .29 \\
\hline 1,000 & 455 & 0.4 & 433 & 0.3 & -22 & -0.1 & 15.81 & .001 & 0.06 & .98 \\
\hline
\end{tabular}

Note-For all $F$ values, $d f=(1,44)$. 
and $374 \mathrm{msec}$ for manual and saccadic RT respectively)] and cued-uncued $[F(1,44)=10.06, p<.003(417$ and $407 \mathrm{msec}$ for cued and uncued, respectively)]. There were three significant interactions. First, response interacted with SOA $[F(1,44)=4.25, p<.05]$, with saccadic RTs increasing at the long SOA and manual RTs decreasing at the long SOA. The second interaction was between response and cued-uncued $[F(1,44)=13.05, p<.001]$, with saccadic responses showing an overall inhibitory effect $(-20 \mathrm{msec})$ and manual responses showing no net cuing effect $(1 \mathrm{msec})$. Finally, the interaction between SOA and cued-uncued was significant $[F(1,44)=49.35, p<.001]$, with the expected net facilitation at the short SOA $(9 \mathrm{msec})$ combined with inhibition at the long SOA $(-29 \mathrm{msec})$. The higher order interaction of response, SOA, and cued -uncued was not significant $[F(1,44)=2.02, p>.16]$.

Planned contrasts revealed that, for manual responses, there was facilitation $(24 \mathrm{msec})$ for cued positions at the short $\operatorname{SOA}[F(1,44)=19.97, p<.001]$ and inhibition $(-22 \mathrm{msec})$ at the long SOA $[F(1,44)=15.81, p<.001]$. For saccadic responses, on the other hand, there was a nonsignificant trend toward inhibition $(-5 \mathrm{msec})$, at the short SOA $[F(1,44)<1, p>.32]$ but significant inhibition $(-36 \mathrm{msec})$ at the long SOA $[F(1,44)=43.40, p<.001]$.

Errors. Analysis of error rates revealed a significant main effect of response $[F(1,44)=5.58, p<.03]$, with more errors for saccadic responses than for manual responses $(1.3 \%$ vs. $0.3 \%)$. There was also an interaction of response and SOA $[F(1,44)=5.85, p<.02]$. The nature of this interaction was such that errors with saccadic responses declined as SOA increased $(3.2 \%$ vs. $0.8 \%)$, whereas errors for manual responses remained unaffected $(0.3 \%$ vs. $0.4 \%)$. The higher order interaction of response, SOA, and cued-uncued was not significant $[F(1,44)=$ $1.88, p>.17]$.

Planned contrasts revealed only one significant effect of cued-uncued; saccadic responses showed a facilitation effect at the short SOA $(1.5 \%)[F(1,44)=7.93, p<.01]$. Note that this facilitation effect is in the opposite direction to the nonsignificant trend observed with RT.

\section{Discussion}

Our results suggest a stronger dissociation than that found by Maylor (1985) between manual and saccadic responses in an IOR paradigm. Manual responses showed early facilitation and late inhibition, whereas saccades, if anything, tended toward nonsignificant inhibition at the short SOA (as was the case in Experiment 1). However, the 5-msec inhibitory effect for saccadic responses at $133 \mathrm{msec}$ may be offset by the $1.5 \%$ facilitatory effect shown in accuracy. Nevertheless, it is clear that, at an SOA of $133 \mathrm{msec}$, saccadic localization responses do not show the strong facilitation observed with manual localization responses.

\section{EXPERIMENT 3}

We have found results analogous to those reported by Maylor (1985)-- specifically, a difference in cuing effects for saccadic and manual responses. This difference occurs in the short-SOA condition, with manual responses being facilitated and saccades (if anything) being inhibited. We believe that there is an initial facilitation following exogenous cues that develops into inhibition for both manual and saccadic responses but that this process is shifted in time for saccades relative to manual responses. Hence, our failure to observe early facilitation for saccadic responses in Experiment 2 (or the cue-back condition of Experiment 1) could be due to our failure to use SOAs shorter than $133 \mathrm{msec}$. If this is the case, one might expect that when a sufficiently broad range of SOAs is used, there should be early facilitation for both manual and saccadic responses. To test this hypothesis, in Experiment 3, we (1) attempted to demonstrate that facilitation of response does occur at short SOAs for saccades and (2) explored more fully the time course of IOR with manual versus saccadic responses.

Furthermore, to rule out the possibility that the dissociation between manual and saccadic responses observed in Experiment 2 was due merely to the fact that different groups of subjects were tested in the two conditions, we used a within-subjects design in Experiment 3.

\section{Method}

Subjects. Twenty undergraduates from Rutgers, Newark, participated and received partial course credit.

Procedure. Each subject was tested in four blocks of 120 trials each, two each for manual responses and for saccadic responses. The subjects completed the two saccadic blocks prior to the manual blocks, or vice versa: The order was counterbalanced between subjects. The procedure for each trial was essentially the same as that followed in Experiment 2, with the major change being that five different SOAs between cue and target were used $(67,94,133$, 200 , and $1,000 \mathrm{msec}$ ). Additionally, the boxes flanking the central fixation point were moved slightly farther out $\left(5.8^{\circ}\right)$. Each combination of cue position (cued-uncued) and SOA was represented by 24 trials. A practice block of 16 trials was completed before starting either the saccadic task or the manual task.

In order to reduce the minimal SOA available to $67 \mathrm{msec}$, the durations of the initial cue brightening was changed to $27 \mathrm{msec}$, the intercue ISI was $13 \mathrm{msec}$, and the fixation brightening was $27 \mathrm{msec}$. We used fixation-target ISIs of $0,27,67,133$, and 933 msec to provide the SOA range reported above (note change from the values indicated in Figure 1).

The sampling rate of the ISCAN eye-tracking system was increased from $60 \mathrm{~Hz}$ to $180 \mathrm{~Hz}$, resulting in the eye position being sampled approximately every $6 \mathrm{msec}$. The higher sampling rate made it possible to switch to a velocity-based criterion for detecting saccades. ${ }^{2}$ During the 1,000-msec period prior to target onset the subjects had to maintain fixation. If the subjects moved their eyes during this period such that eye movement velocity went above $100^{\circ} /$ second or their eyes moved farther than $2.5^{\circ}$ from fixation, it was assumed that they had made a saccade, and the trial was terminated and replaced in the pool of uncompleted trials.

After target onset, a saccade was assumed to have started when eye velocity exceeded $100 \%$ second, and the end of the saccade was indicated when velocity fell below $12^{\circ}$ second. The terminal point of the saccade had to be within $4.4^{\circ}$ of the target position to be scored as correct. The criteria were essentially the same for manual responses, with the exception that the velocity of the cursor controlled by the mouse was measured. The cutoff velocities for the start and end of manual responses were $24 \%$ second and $12 \%$ second, respectively. 


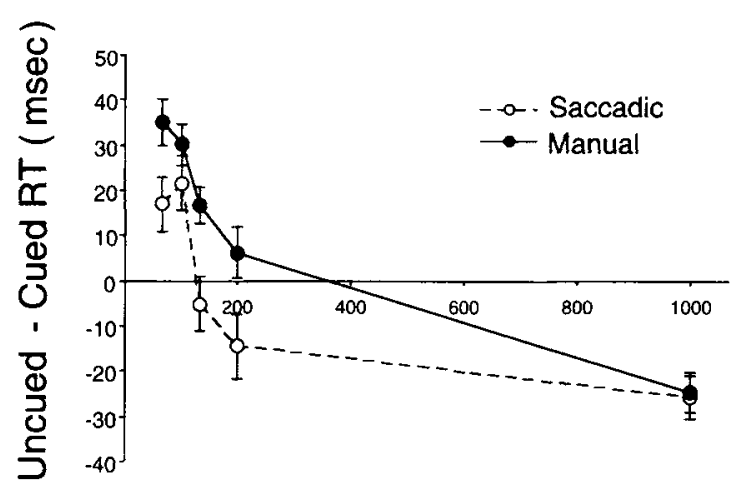

\section{SOA (msec)}

Figure 3. Experiment 3, cuing effect (uncued RT - cued RT) as a function of SOA and response mode (saccadic or manual). Note the contrasting pattern (facilitation vs. inhibition) for manual and saccadic responses in the range $133-200 \mathrm{msec}$.

\section{Results}

Trimming procedures (i.e., slow or fast responses, blinks, and anticipations) resulted in exclusion of $4.1 \%$ of the data. Difference scores (uncued RT - cued RT) for the manual and saccadic tasks across the five SOAs tested are shown in Figure 3 (see also Table 3 ).

RT. As can be seen in Figure 3 and Table 3, the manual and saccadic versions of this task showed a differing time course. Both responses showed initial facilitation and later IOR. However, the transition point from facilitation to inhibition differed for the two responses, occurring earlier for saccadic responses. This pattern was confirmed by ANOVA and a series of planned contrasts.

All effects and interactions except for the highest order interaction were statistically significant in an ANOVA with response, SOA, and cued-uncued. Responses were much faster for saccades than for the mouse ( $289 \mathrm{vs.}$ $369 \mathrm{msec})[F(1,19)=126.73, p<.001]$. SOA was significant $[F(4,76)=23.10, p<.001]$, with a clear increase in RT at the longest SOA $(328,321,319,322$, and $354 \mathrm{msec}$ for the shortest to longest SOAs). Finally, cued-uncued showed overall facilitation on cued trials relative to on uncued trials $(6 \mathrm{msec})[F(1,19)=5.75, p<.03]$. The interaction between response and SOA was significant $[F(4,76)=13.17, p<.001]$, as was that between response and cued-uncued $[F(1,19)=10.28, p<.005]$. This latter interaction showed that manual responses were facilitated by cues $(13 \mathrm{msec})$, whereas saccadic responses showed no overall effect of cue $(-1 \mathrm{msec})$. The interaction between cued-uncued and $\operatorname{SOA}[F(4,76)=36.49$, $p<.001]$ showed that the cues facilitated responses at the three shortest SOAs $(26,26$, and $5 \mathrm{msec})$ but inhibited responses at the two longer SOAs $(-4$ and $-25 \mathrm{msec})$. The higher order interaction of response, cued-uncued, and SOA fell short of significance $[F(4,76)=1.84, p>$ .13].

The strength of the cuing effects for manual and saccadic responses was calculated separately for each of the five SOAs. The results are displayed in Table 3 and are indicated graphically in Figure 3 as well. The gist of these analyses was that manual responses were facilitated following cues at SOAs of 67 through $133 \mathrm{msec}$, with a nonsignificant trend toward facilitation at $200 \mathrm{msec}$ and strong inhibition following cues with an SOA of $1,000 \mathrm{msec}$. For saccadic responses, facilitation was found only at the two shortest SOAs. There was a nonsignificant trend toward inhibition at the $133-\mathrm{msec}$ SOA and significant inhibition at SOAs of 200 and $1,000 \mathrm{msec}$.

Planned contrasts were also used to compare the size of the cuing effects for manual and saccadic responses. At the shortest $(67 \mathrm{msec}) \mathrm{SOA}$, manual responses showed more facilitation than did saccadic responses $[F(1,19)=$ $7.67, p<.02]$, but this difference was eliminated by $94 \mathrm{msec}[F(1,19)=1.67, p>.21]$. Manual responses again differed from saccadic responses at the intermediate $133 \mathrm{msec}$ SOA $[F(1,19)=11.08, p<.004]$ and 200 msec SOA $[F(1,19)=9.95, p<.006]$. Finally, the amount of IOR for the two responses at $1,000 \mathrm{msec}$ did not differ $(F<1)$.

Errors. As was the case with the RT analysis, there were a number of significant effects when errors were analyzed. SOA affected error rates $(1.9 \%, 1.6 \%, 2.0 \%$, $1.5 \%$, and $0.4 \%$ for shortest to longest SOAs) $[F(4,76)=$ $4.05, p<.005]$. There were also more errors on uncued trials than on cued trials $(2.5 \%$ vs. $0.5 \%)[F(1,19)=$ $21.68, p<.001]$. The only interaction to reach significance was that between SOA and cued-uncued $[F(4,76)=$ $3.40, p<.02]$. Facilitation effects for errors tended to decrease with SOA, with the greatest facilitation at $67 \mathrm{msec}(3.2 \%)$ and the smallest at $1,000 \mathrm{msec}(0.5 \%)$. Finally, the interaction of response, cued-uncued, and SOA was not significant $[F(4,76)=1.57, p>.18]$.

The results of the planned contrasts on cuing effects for errors are shown in Table 3, and the findings are basically in agreement with the corresponding RT data. For manual responses, cues resulted in significant facilitation at each of the four shortest SOAs. This is entirely compatible with the trend toward facilitated RT in these conditions.

For saccadic responses, there were only two significant cuing effects for errors. First, cues lead to facilitation at the 67-msec SOA (where RT was, of course, also facilitated). Similarly, a significant decrease in errors was observed following spatial cues at the 133-msec SOA, in contrast with the inhibition effect observed in this condition for RT (i.e., cued RT is slower).

\section{Discussion}

Whether one considers the RT or error data, it is clear that facilitation and IOR effects for manual and saccadic localization responses do not follow the same time course. In RT, manual responses were facilitated up to an SOA of $133 \mathrm{msec}$, and significant IOR did not develop until the longest SOA tested. For saccadic responses, there was facilitation only up to $94 \mathrm{msec}$, whereas IOR developed by $200 \mathrm{msec}$. Considering the error data, 
Table 3

\begin{tabular}{|c|c|c|c|c|c|c|c|c|c|c|}
\hline \multirow{2}{*}{$\begin{array}{c}\text { SOA } \\
(\mathrm{msec}) \\
\end{array}$} & \multicolumn{2}{|c|}{ Cued } & \multicolumn{2}{|c|}{ Uncued } & \multicolumn{2}{|c|}{ Uncued - Cued } & \multicolumn{2}{|c|}{ RT } & \multicolumn{2}{|c|}{$\%$ Error } \\
\hline & RT & $\%$ Error & $\mathrm{RT}$ & $\%$ Error & RT & $\%$ Error & $F$ & $p<$ & $F$ & $p<$ \\
\hline \multicolumn{11}{|c|}{ Saccadic Responses } \\
\hline 67 & 275 & 0.5 & 292 & 4.1 & 17 & 3.6 & 13.49 & .001 & 26.47 & .001 \\
\hline 94 & 268 & 1.3 & 290 & & 22 & 1. & 21.79 & .001 & 2.74 & .11 \\
\hline 133 & 274 & 0.7 & 269 & 4.8 & -5 & 4.1 & 1.18 & .29 & 34.23 & .001 \\
\hline 200 & 289 & 1.1 & 275 & 2.3 & -14 & 1.2 & 9.75 & .003 & 2.84 & .10 \\
\hline 1,000 & 339 & 0.2 & 313 & 0.9 & -26 & -0.7 & 31.98 & .001 & 1.01 & .32 \\
\hline \multicolumn{11}{|c|}{ Manual Responses } \\
\hline 67 & 354 & 0.3 & 389 & 3.0 & 35 & 2.7 & 57.58 & .001 & 15.28 & .001 \\
\hline 94 & 349 & 0.2 & 379 & 2.3 & 30 & 2.1 & 42.26 & .001 & 9.05 & .004 \\
\hline 133 & 358 & 0.2 & 375 & 2.3 & 17 & 2.1 & 13.09 & .001 & 9.13 & .004 \\
\hline 200 & 359 & 0.2 & 365 & 2.3 & 6 & 2.1 & 1.79 & .19 & 8.98 & .004 \\
\hline 1,000 & 394 & 0.0 & 369 & 0.4 & -25 & -0.4 & 28.26 & .001 & $<1$ & .57 \\
\hline
\end{tabular}

Note-For all $F$ values, $d f=(1,76)$.

manual responses were facilitated up to $200 \mathrm{msec}$, whereas saccades show facilitation only up to $133 \mathrm{msec}$. It is also worth pointing out that the IOR effects observed with manual and saccadic responses are of comparable magnitude.

The major conclusions from Experiments 2 and 3 are threefold. First, as just pointed out, IOR develops more quickly for saccadic responses than for manual responses. Second, both manual and saccadic responses show facilitation at short SOAs. This conclusion differs from that reached by Maylor (1985), who never succeeded in showing any early facilitation for her saccadic localization task. The third conclusion from Experiments 2 and 3 , which also differs from that of Maylor, is that the amount of IOR obtained for saccadic and manual responses was equivalent. Maylor reported that saccadic responses resulted in a larger amount of IOR, but she tested only up to an SOA of $500 \mathrm{msec}$. Given the pattern observed in Experiment 3 (see Figure 3), it would seem that her conclusion of greater IOR for saccadic responses may apply only to intermediate SOAs, where IOR for manual responses has not yet had time to reach a maximum.

\section{EXPERIMENT 4}

Experiment 4 involved only manual responses. In Maylor's (1985) study, the manual response involved keypress (left vs. right), which could be argued differs significantly from the less abstract spatial mapping involved in the "mouse" version of the manual task used here. We thus used a version of the manual localization task more comparable to the one Maylor carried out. In Experiment 4, subjects performed two versions of the manual target localization task (mouse and keypress). Our goal was to determine whether the difference be- tween her results and the present findings was in part due to the nature of the manual response that we used.

\section{Method}

Subjects. Fourteen Rutgers, Newark, undergraduates participated in a single 20 -min session and received partial course credit.

Procedure. Each subject was tested in two versions of the localization task ( 240 trials each), preceded by a 16-trial practice block. The "mouse" version of the localization task was identical to the manual task used in Experiment 3. In the "keys" version of this task, the subjects used the " 1 " and "2" keys on a computer keyboard to indicate whether the target was located in the left or right display position. The subjects responded with the preferred hand and pressed the space bar with the other hand to initiate each trial. A millisecond timer was used to measure response latency for this task.

\section{Results}

Elimination of fast and slow responses accounted for less than $1 \%$ of all trials.

RT. Cuing effects are shown in Figure 4 (see also Table 4). The overall pattern suggests that the two different manual responses (keypress vs. mouse) followed a similar time course in terms of cuing effects. In particular, the transition point from facilitatory to inhibitory effects appeared to occur at around the same cue-target SOA. An ANOVA with response, SOA, and cued-uncued was performed. All three main effects were significant. Mouse responses were faster than were keypress responses (386 vs. $468 \mathrm{msec}$ ) $[F(1,13)=19.50, p<.001]$. SOA was also significant $(437,432,426,417$, and $424 \mathrm{msec}$ for the shortest to longest SOAs) $[F(4,52)=$ $4.16, p<.006]$. Finally, cued--uncued was significant $[F(1,13)=11.12, p<.006]$, with faster responses on cued trials than on uncued trials ( $420 \mathrm{vs.} 434 \mathrm{msec}$ ). The only interaction to reach significance was that between cued-uncued and SOA $[F(1,13)=24.43, p<.001]$. As Figure 4 makes clear, there was facilitation of re- 


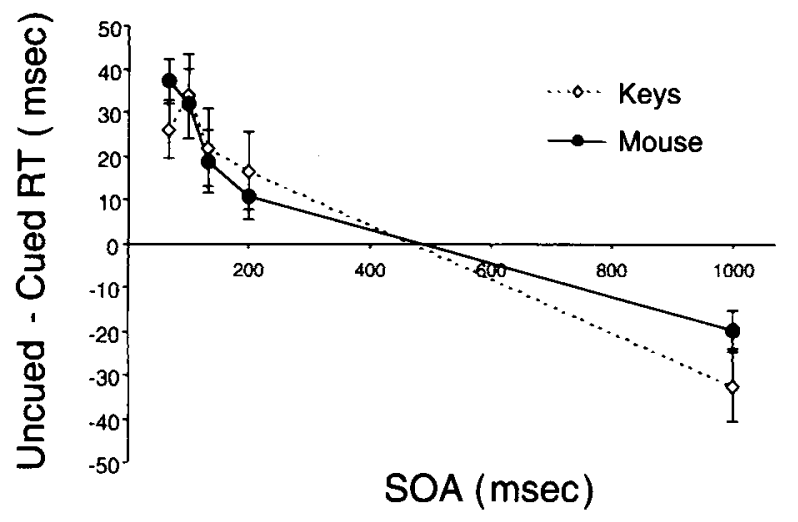

Figure 4. Experiment 4, cuing effect (uncued RT - cued RT) as a function of SOA and type of manual response (mouse or keys).

sponses following cues at the four shortest SOAs, but there was inhibition at the longest SOA. The interaction of response, cue position, and SOA was not significant $[F(1,13)=1.51]$.

Table 4 shows the cuing effects for both modes of response, for each SOA. Also presented are the $F$ and $p$ values for the 10 contrasts tested. All of the cuing effects were significant, and the pattern is clear; for both mouse and keypress responses, there was facilitation for the four shortest SOAs, and there was IOR for the $1,000-\mathrm{msec}$ SOA.

Errors. Analysis of the error rates revealed two significant main effects. The first effect was that of SOA $[F(4,52)=2.76, p<.04]$. The second was that of cueduncued $[F(1,13)=12.93, p<.004]$, with more errors on uncued trials. There were also two significant interactions. The first was that between response type and SOA $[F(4,52)=3.15, p<.03]$ (mouse responses had the lowest error rate at the longest SOA, whereas keypress responses had the lowest error rate at the shortest SOA). Finally, the interaction of response type, SOA, and cued-uncued was significant $[F(4,52)=2.58, p<.05]$.

The planned contrasts on the error data (see Table 4) supported the prior interpretation of the RT data. In the comparisons where cued-uncued affected error rates, the direction of the effect was consistent with the analogous comparison using the RT data.

\section{Discussion}

Despite the fact that the two versions of the manual task likely involved different response requirements (and in fact differed substantially in overall latency), they both showed the same pattern of effects: Facilitation was obtained until at least an SOA of $200 \mathrm{msec}$. At this same SOA interval, however, saccadic responses were inhibited at cued locations (see Experiment 3). As expected, IOR was obtained for both types of manual responses at the $1,000 \mathrm{msec}-\mathrm{SOA}$.

\section{CONCLUSIONS}

In the present experiments, we examined the time course of spatial attention effects in manual and saccadic response systems. There were three main findings. First, there was early facilitation of both saccadic and manual responses. While saccadic facilitation developed somewhat more slowly, it became statistically equivalent to manual facilitation by an SOA of $94 \mathrm{msec}$. Second, IOR was equivalent for the two responses. Finally, and most significantly, manual and saccadic responses differed in the time course over which the initial facilitation effect developed into IOR. Facilitation appeared to decline more quickly, and IOR appeared to develop sooner, for saccadic responses than for manual responses. We first consider each of these points in more detail and then discuss possible explanations for our results.

Table 4

Mean Response Times (in Milliseconds), Percent Errors, and Cue Position Effects (Uncued - Cued) in Experiment 4 as a Function of Response Type and SOA, Along With the $F$ Values for Planned Contrasts Used to Assess Cuing Effects and the Probability ( $p$ ) Levels Associated With Those Particular Contrasts

\begin{tabular}{|c|c|c|c|c|c|c|c|c|c|c|}
\hline \multirow{3}{*}{$\begin{array}{c}\text { SOA } \\
(\mathrm{msec})\end{array}$} & \multirow{2}{*}{\multicolumn{2}{|c|}{ Cued }} & \multirow{2}{*}{\multicolumn{2}{|c|}{ Uncued }} & \multirow{2}{*}{\multicolumn{2}{|c|}{ Uncued - Cued }} & \multicolumn{4}{|c|}{ Planned Contrasts } \\
\hline & & & & & & & \multicolumn{2}{|c|}{$\mathrm{RT}$} & \multicolumn{2}{|c|}{$\%$ Error } \\
\hline & RT & $\%$ Error & RT & $\%$ Error & RT & $\%$ Error & $F$ & $p<$ & $F$ & $p<$ \\
\hline \multicolumn{11}{|c|}{ Mouse Responses } \\
\hline 67 & 375 & 0.6 & $4 ! 2$ & 3.9 & 37 & 3.3 & 55.51 & .001 & 16.21 & .002 \\
\hline 94 & 371 & 0.3 & 403 & 1.8 & 32 & 1.5 & 41.21 & .001 & 3.21 & .08 \\
\hline 133 & 375 & 0.9 & 394 & 3.6 & 19 & 2.7 & 14.09 & .001 & 10.61 & .002 \\
\hline 200 & 373 & 1.0 & 384 & 4.0 & 11 & 3.0 & 4.81 & .04 & 10.36 & .002 \\
\hline 1,000 & 396 & 0.3 & 377 & 0.0 & -19 & -0.3 & 15.53 & .001 & $<1$ & .72 \\
\hline \multicolumn{11}{|c|}{ Keypress Responses } \\
\hline 67 & 467 & 0.0 & 493 & 0.9 & 26 & 0.9 & 27.05 & .001 & 1.21 & .28 \\
\hline 94 & 460 & 0.3 & 494 & 2.4 & 34 & 2.1 & 45.74 & .001 & 6.42 & .02 \\
\hline 133 & 457 & 1.5 & 479 & 3.3 & 22 & 1.8 & 19.09 & .001 & 4.49 & .04 \\
\hline 200 & 448 & 1.5 & 464 & 1.2 & 16 & -0.3 & 10.95 & .002 & $<1$ & .69 \\
\hline 1,000 & 478 & 0.9 & 445 & 1.5 & -33 & 0.6 & 43.06 & .001 & $<1$ & .45 \\
\hline
\end{tabular}




\section{Magnitude of Facilitation and IOR for Manual Versus Saccadic Responses}

The literature contains only one study that directly addressed the relative time course of visual attention effects for manual and saccadic responses, that by Maylor (1985). Maylor compared facilitation and inhibition in response to exogenous cues for manual and saccadic tocalization responses. She tested SOAs in the range of 100-500 msec and reported that (1) saccadic responses were not facilitated at the shortest SOA, whereas manual responses were, and (2) saccadic responses showed greater IOR at the 500-msec SOA. She interpreted the different pattern of manual and saccadic data to be due to some type of inhibitory mechanism that prevented reflexive eye movements to the peripheral cues. This could be conceived of as a monotonic increase in saccadic RT following cued trials, which shifts the cued RT function relative to uncued RTs. The net result of such an increase would be that, for saccadic responses, any decrease in facilitation following spatial cues at short SOAs would be matched by corresponding increases in IOR at longer SOAs, of approximately the same magnitude.

However, we report findings that are at odds with this interpretation. While saccades did show less facilitation at the shortest SOA tested $(67 \mathrm{msec})$, this difference was eliminated by $94 \mathrm{msec}$. We also observed equivalent levels of IOR for the two responses at a 1,000-msec SOA. Thus, there is no evidence for any type of "monotonic delay" of saccadic responses following spatial cues. Instead, it appears that manual and saccadic localization responses show similar levels of facilitation and IOR if more extreme ranges of SOA are sampled. Put another way, saccadic and manual responses show similar patterns if performance is measured either (1) at the peak of facilitation before IOR has started to develop or (2) after IOR has had time to develop fully. The inhibitory effect on saccadic responses following exogenous cues postulated by Maylor (1985) might account for the decreased facilitation for saccades at $67 \mathrm{msec}$, but this would require that the inhibition last for only a very brief period (less than $100 \mathrm{msec}$ ).

\section{Transition from Facilitation to IOR}

The most important observation from our experiments concerns the rate of development of IOR. While both manual and saccadic responses develop similar levels of facilitation prior to IOR, the point at which facilitation starts to develop into IOR differs for the two response modes. For manual localization responses (both mouse and keypress), facilitation extends up to an SOA of at least $200 \mathrm{msec}$. Presumably, it also persists somewhat beyond this point, although our experiments cannot narrow down the precise transition point. However saccadic responses showed significant IOR by the same $200-\mathrm{msec}$ SOA and a trend toward IOR sooner than this. Thus, there is a range of cue-target SOAs (roughly $133-200 \mathrm{msec}$ or more) wherein saccadic localization responses are in- hibited by spatial cues, whereas manual localization response to the same cues are facilitated.

\section{Explanation of the Differing Time Course}

The core finding of the present study is the fact that, at certain cue-target intervals, one can observe either facilitation or inhibition, depending on the nature of the response. We will consider five possible explanations that might be offered to account for the present findings.

1. Standard model. To a first approximation, the present data appear difficult to reconcile with the "standard model" described in the introduction. If we assume that the spatial attention system is separable from other "data processing" systems, then presumably the cognitiveneural systems responsible for stimulus evaluation and response selection act on information provided them by the spatial attention system (which obviously might itself involve numerous neural pathways). In a target localization task, spatial cues should facilitate encoding, detection, or evaluation of targets appearing at cued locations. This in turn should develop into IOR as the spatial map or representation coding the position of the spatial cue gets tagged to prevent attention from returning. However, there appears to be no simple or plausible mechanism within this framework that would allow for manual and saccadic localization responses to have different time courses, as was observed here.

2. Task difficulty. There is evidence in the literature that differences in the time course of spatial cuing and IOR effects can arise even when the same response modality is used (Lupiáñez, Milán, Tornay, Madrid, \& Tudela, 1997; see also Terry, Valdes, \& Neill, 1994). Lupiáñez et al. compared the time course of cuing effects for detection and discrimination tasks and found that IOR developed sooner for detection than for discrimination. A possible reason for this finding could have been that the discrimination task was more attentionally demanding than the relatively easy detection task. Thus, it is possible that the more cognitively demanding a task is, the more persistent facilitation will be before developing into IOR.

We do not believe that this suggestion provides a reasonable explanation for the present findings. Both our manual and saccadic tasks required target localization, and these tasks were specifically chosen to reduce, if not eliminate, any differences in task difficulty between the two response modes. One might argue that simply looking at the target stimulus is less cognitively demanding than using a mouse to control a cursor on a display screen (albeit that they both should be less demanding than a task requiring discrimination between target stimuli). However, one might just as easily maintain that pressing left or right response keys should likewise be a more abstract and complex task than using the mouse. Yet, in Experiment 4 , there was absolutely no evidence that IOR developed at different rates for the two manual responses. Indeed, a comparison of overall response latencies for the various tasks in Experiments 3 and 4 shows no rela- 
tionship between overall RT (a possible index of task difficulty) and the rate of development of IOR. Thus, while present evidence does not allow us to rule out some version of this explanation, we do not consider it a particularly attractive alternative.

3. Separate facilitatory and inhibitory components of cuing. One interesting possibility that cannot be ruled out based on the present data alone was proposed in an article by Tipper et al. (1997; see also Weaver, Lupiáñez, \& Watson, 1998). Tipper et al. (1997) suggested that an exogenous spatial cue leads to separate facilitatory and inhibitory effects, which each have a different time course. What is observed empirically as the onset of IOR is the result of the combined effect of a decaying facilitatory component and a slower rising but more persistent inhibitory component. Although the neural pathways responsible for these components were not directly specified, Tipper et al. (1997) reported evidence suggesting that facilitation was mediated by subcortical systems, whereas inhibition was mediated cortically.

Such a proposed framework might potentially be capable of accounting for the present results, provided that certain assumptions are allowed. Since IOR develops more quickly for saccadic responses than for manual responses, one possibility is that the facilitatory component decays more quickly or is less robust for saccades. This may explain the present results, since one would then expect the combined effects of facilitation and inhibition to cause net inhibition (i.e., IOR) at shorter cue-target SOAs. Indeed, the initial facilitation effect for saccades was smaller than that for manual responses (see Experiment 3). However, if the differing time course for the two responses were due merely to a lower baseline for a facilitation component of saccades, one would have expected that the cuing effect for saccades should have continued to decline after the 67-msec SOA, which did not occur. In fact, the difference in amount of facilitation for manual and saccadic responses was reduced and nonsignificant at the 94msec SOA.

Alternatively, suppose that the inhibitory component has a faster rise time or is more pronounced for saccades. This too would lead to an earlier transition to IOR, even if baseline facilitation was equivalent for manual and saccadic responses. An increased inhibitory mechanism for saccades might act to offset any tendency for exogenous visual stimuli to reflexively attract orienting responses from the saccadic system. ${ }^{3}$

The question of whether or not these dual processes exist, however, seems orthogonal to the issue at hand. That is, there seems to be nothing inconsistent with the basic tenets of premotor theory in suggesting that separate facilitatory and inhibitory attentional effects can be exerted on spatiomotor maps and, furthermore, that these effects can be different for manual and saccadic responses. Thus, the proposition that separate facilitatory and inhibitory processes occur following an exogenous cue should not be considered as ruling out some form of premotor theory (or response-based models in general) as explanations of IOR. These putative dual processes could be instantiated within either a response-based or attentionbased (standard model) explanation of IOR. ${ }^{4}$

4. Modality-specific effects of fixation brightening. Could the differential time course for manual and saccadic responses be due to some modality-specific characteristic of the cue-back procedure? It has been shown that fixation neurons in the superior colliculus, which have foveal receptive fields, will inhibit eye movements following stimulation (Munoz \& Wurtz, 1995). Thus, the cue-back procedure, where the fixation point brightens prior to target onset, might have inadvertently affected saccadic performance, leaving manual responses unaffected. The differential time course would thus have occurred because saccades were (at least for the shorter SOAs) inhibited relative to manual responses.

However, a systematic slowing of saccade latencies should affect both cued and uncued trials. Hence, such an explanation would have no effect on cuing effects (which represent RT differences) and could not account for the attentional differences between saccades and manual responses reported here.

5. Premotor theory. Premotor theory maintains that there is no need to postulate an anatomically separate spatial attention system; the physiological maps that are involved in spatial attention are also involved in sensory processing and response preparation and/or execution. As previously stated, this approach assumes that the act of attending to a spatial location is nothing more or less than the act of preparing to execute some type of response to that location. Given these assumptions, three facts about the neural representation of space take on great significance. First is the observation that there is no single cortical map used to represent space but rather that there are many such maps. Second, the cortical areas used to represent space code not only for specific sensory events but also for specific motor responses as well. Finally, these various maps differ in the type of response they code for. Thus, a given neuron may be active when a saccade is to be made to a stimulus location, whereas another may fire only when a reaching or grasping response is required.

Although not specifically predicting such, premotor theory clearly allows for the possibility that different spatiomotor maps will have different properties regarding attentional effects. The typical reaching response is a much more complicated behavior than is an eye movement (Robinson, 1981). Hence, such differences are likely to be represented in the organization or even the number of their respective spatiomotor maps. Whether these differences will result in differences in their attentional effects remains an empirical question. For our purposes, the primary question was whether putative differences in these maps could lead to differences in the magnitude and/or time course of facilitation or IOR effects for manual and saccadic localization responses. On the basis of the results from the present experiments, it would appear that the time courses do differ; both manual and saccadic localization responses are initially facilitated by roughly 
the same amount, but IOR develops more quickly for saccades than for manual responses. Indeed, at some range of cue-target SOAs, the spatiomotor map coding for eye movements is inhibited, whereas the spatiomotor map coding for manual responses is facilitated.

There are now a number of reports indicating that IOR can be observed in discrimination tasks (Cheal, Chastain, \& Lyon, 1998; Lupiáñez \& Solano, 1997; Pratt, 1995; Pratt et al., 1997). Some would argue that the observation of IOR effects in discrimination tasks casts doubt on response-based explanations of IOR (but see Taylor \& Klein, 1998, for counterarguments). However, recent reports of both shape and object selectivity in eyemovement and hand-movement spatial maps in parietal cortex (Sereno \& Maunsell, 1998, and Murata, Gallese, Kaseda, \& Sakata, 1996, respectively) would suggest that premotor theory may also be able to account for such IOR effects in discrimination tasks, as well as object-based IOR. In any event, the present data suggest that any model of IOR will need to account for response-based effects such as those reported here.

\section{REFERENCES}

ABRAMs, R. A.. \& DOBRiN, R. S. (1994). Inhibition of return: Effects of attentional cuing on eye movement latencies. Journal of Experimental Psichology: Human Perception \& Performance, 20, 467-477.

Chrat. M., Chasiain, G.. \& Lyon. D. R. (1998). Inhibition of return in visual identification tasks. Visual Cognition. 5, 365-388.

GiRSoN, B. S. \& E(il:TH. H. (1994). Inhibition of return to object-based and environment-based locations. Perception \& Psuchophwics, 55. 323-339.

GoomAl.F. M. A.. \& Mil.ni:R. D. (1992). Separate visual pathways for perception and action. Trends in Newrosciences, 15, 20-25.

JORDAN. H. \& TIPPER. S. P. (1998). Object-based inhibition of return in static displays. Psychonomic Bulletin \& Review, 5, 504-509.

KLEIN, R. M. (1988). Inhibitory tagging facilitates visual search. $\mathrm{Na}$ ture. 334, 430-43!

KLEIN. R. M. (2000). Inhibition of return: Who, what, when, where, how, and why. Trends in Cognitive Sciences, 4, 138-147.

KLEIN. R. M.. \& TAY1.oR. T. L. (1994). Categories of cognitive inhibition with reference to attention. In D. Dagenbach \& T. H. Carr (Ed.), Inhibitory processes in att'ntion, memory, and language (pp. 113150). San Diego: Academic Press.

KUsTov. A. A.. \& RoBINSON. D. L. (1996). Shared neural control of attentional shifts and eye movements. Nahure, 384, 74-77.

Lupí́ñez. J., Mil.án, E. G., Tornay, F. J.. Madrid, E. \& Tudel.a, R (1997). Does IOR occur in discrimination tasks? Yes, it does, but later. Perception \& Psychophsics, 59, 1241-1254.

LUPIÁÑ̇.Z. J.. \& SOI.ANO, C. (1997). Inhibition of return in a colour discrimination task: No interaction with the Simon effect. Cognitiva, 9. 195-205.

MAYLOR, E. A. (1985). Facilitatory and inhibitory components of orienting in visual space. In M. 1. Posner \& O. S. M. Marin (Eds.), Attention and performance XI (pp. 189-204). Hillsdale, NJ: Erlbaum

Mondor, T. A.. Brfau. L. M., \& Milliken. B. (1998). Inhibitory processes in auditory selective attention: Evidence of location-based and frequency-based inhibition of return. Perception \& Psychophvsics. 60, 296-302.

MuNoz, D., \& WuRrz. R. H. (1995). Saccade-related activity in monkey superior colliculus: Il. Spread of activity during saccades. Jominal of Neurophysiologry, 73, 2334-2348.

Murata. A., Gailese, V., Kaseda. M.. \& Sakata, H. (1996). Parietal neurons related to memory-guided hand manipulation. Journut of Neurophrsiologr: 75, 2180-2186.
Posner, M. I., \& Cohen. Y. A. (1980, November). Consequences of visual orienting. Paper presented at the annual meeting of the Psychonomic Society, St. Louis.

Posner, M. I., \& COHEN, Y. A. (1984). Components of visual orienting. In H. Bouma \& D. G. Bouwhuis (Eds.), Attention and performance $X$ (pp. 531-556). Hillsdale, N]: Erlbaum.

Posner, M. I., Rafal, R. D., Choate, L. S.. \& Vaughan, J. (1985). Inhibition of return: Neural basis and function. Cognitive Neuropsychologi, 2, 211-228.

PRATT, J. (1995). Inhibition of return in a discrimination task. Psychonomic Bulletin \& Revien, 2, 117-120.

Pratt. J.. Kincistone, A., \& KhOE, W. (1997). Inhibition of return in location- and identity-based choice decisions. Perception \& Psychophysics, 59, 964-971.

Rafal. R. D., Cal.abresi, P. A., Brennan, C. W. \& Sciolto, T. K (1989). Saccade preparation inhibits reorienting to recently attended locations. Joumul of Experimental Psychology: Human Perception \& Performance, 15, 673-685.

Rafai, R. D. Egly, R., \& RHodes, D. (1994). Effects of inhibition of return on voluntary and visually guided saccades. Canadian Journal of Experimental Psychologv. 48, 284-300.

Reuter-Lorenz, P. A.. Jha. A. P., \& Rosenquist, J. N. (1996). What is inhibited in inhibition of return? Journal of Experimental Psychology: Human Perception \& Performance, 22, 367-378.

Rizzolat TI, G.. Rigiglo. L., Dascola, l., \& Umiltà, C. (1987). Reorienting attention across the horizontal and vertical meridians: Evidence in favor of a premotor theory of attention. Neuropsychologia, $25,31-40$.

Rizzolat'i, G., Riggio, L., \& Sheliga, B. M. (1994). Space and selective attention. In C. Umiltà \& M. Moscovitch (Eds.), Attention and performance $X V$ (pp. 231-265). Cambridge, MA: MIT Press.

RoBINSon, D. A. (1981). Control of eye movements. In V. B. Brooks (Ed.). Handhook of phvsiologv: Section 1. The nervous svstem: Vol. II. Motor control: Part 2 (pp. 1275-1320). Bethesda, MD: American Physiological Society.

SERENO, A. B. (1996). Parsing cognitive processes: Psychopathological and neurophysiological constraints. In S. Matthysse, D. L. Levy, et al. (Eds.), Psychopathology: The evolving science of mental diserder (pp. 407-432). New York: Cambridge University Press.

Serfino. A. B.. \& MaUnsell. J. H. R. (1998). Shape selectivity in primate lateral intraparietal cortex. Nature, 395, 500-503.

SHEPARD, M.. FINDI.AY, J. M., \& HOCKEY, R. J. (1986). The relationship between eye movements and spatial attention. Quarterly Journal of Experimental Psychology, 38A, 475-491.

Shimolo, S., Tanaka, Y., Hikosaka, O., \& Miyauchi, S. (1996). Vision. aftention and action: Inhibition and facilitation in sensorymotor links revealed by the reaction time and the line motion. In T. Inui \& J. L McClelland (Eds.), Attention and performance $X V I$ (pp. 597-630). Cambridge, MA: MIT Press.

TAYIOR. T. L.. \& KLEIN, R. M. (1998). On the causes and effects of inhibition of return. Psychonomic Bulletin \& Review', 5, 625-643.

Terry, K. M.. Valdes, L. A., \& Nelll, W. T. (1994). Does "inhibition of return" occur in discrimination tasks" Perception \& Psychophisics, 55, 279-286.

Tipper. S. P.. Driver, J., \& Weaver, B. (1991). Object-centered inhibition of return of visual attention. Quarterly Journal of Experimental Psuchologu, 43, 289-298.

TIPPER, S. P., JORDAN, H., \& WEAVER, B. (1999). Scene-based and objectcentered inhibition of return: Evidence for dual orienting mechanisms. Perception \& Psychophysics, 61, 50-60.

Tipper, S. P.. Weaver, B., Jerreat, L. M.. \& Burak, A. L. (1994). Object-based and environment-based inhibition of return of visual attention. Journal of Experimental Psvchology: Human Perception \& Performance, 20, 478-499.

Tipper, S. P., Wifaver, B., Rafal., R., Starkvfldt, Y., Ro. T., Egly, R., DANZIGER, S. \& REUTER-LORENZ. P. A. (1997). Object-based facilitation and inhibition from visual orienting in the human split brain. Journal of Experimental Psychology: Human Perception \& Performance, 23, 1522-1532

Uncierleider. L. G.. \& Mishinin. M. (1982). Two cortical visual sys- 
tems. In D. J. Ingle, M. A. Goodale, \& R. J. W. Mansfield (Eds.), Analysis of visual behavior (pp. 549-586). Cambridge, MA: MIT Press.

Weaver, B., Lupiáñez, J., \& WATSON, F. L. (1998). The effects of practice on object-based, location-based, and static-display inhibition of return. Perception \& Psychophysics, 60, 993-1003.

\section{NOTES}

1. Response-based models would seem to explicitly require some functional linkage between eye movements and attention; hence, such evidence has proven useful to advocates of this approach. However, any putative link between eye movements and spatial attention is actually neutral on the issue of whether the standard or some response-based explanation of IOR is more suitable.

2 . The software necessary to measure saccade velocity was not available when the first two experiments were run. Since velocity-based criteria to detect saccades are more frequently used in the literature, we chose to switch to this method in the later experiments. This change in procedure is unlikely to have affected the overall pattern of results. Even if a systematic change in saccade latencies were to result from this change, it would affect every condition equally, leaving the cuing effects (uncued - cued difference) unaffected.

3. This suggestion is somewhat analogous to that offered by Maylor (1985) to explain her data but is actually rather different. Whereas she proposed an inhibition of saccades to cued locations that was superimposed on performance across all SOAs, the suggestion considered here is that inhibition develops only gradually over time, and, thus, its effects may not be apparent at all cue-target intervals.

4. While either model might be adapted to include dual facilitatory and inhibitory effects, that is not to say that each model is equally plausible in this context. In particular, to account for the present data, both models would have to assume (for example) that inhibition developed more quickly for saccades than for manual responses. The possible functional reason for increased inhibition for saccades that was suggested in the previous paragraph strikes us as being rather ad hoc when considered in the context of the standard model of attention, whereas this explanation is arguably more plausible in the context of premotor theory.

(Manuscript received October 13, 1998; revision accepted for publication March 2, 2000.) 